\title{
Present and Future Observations of the Earthshine from Antarctica
}

\author{
Danielle Briot $^{1}$, Luc Arnold ${ }^{2}$ and Stéphane Jacquemoud ${ }^{3}$ \\ ${ }^{1}$ Observatoire de Paris, 61 avenue de l'Observatoire, 75014 Paris, France \\ email: danielle.briot@obspm.fr \\ ${ }^{2}$ Observatoire de Haute-Provence, 04870 Saint-Michel l'Observatoire, France \\ email: luc.arnold@oamp.fr \\ ${ }^{3}$ Université Paris Diderot / Institut de Physique du Globe de Paris \\ 39 rue Hélène Brion, 75013 Paris, France \\ email: jacquemoud@ipgp.fr
}

\begin{abstract}
It is likely that images of Earth-like planets will be obtained in the next years. The first images will actually come down to single dots, in which biomarkers can be searched. Taking the Earth as a example of planet providing life, Earthshine observations showed that the spectral signature of photosynthetic pigments and atmospheric biogenic molecules was detectable, suggesting that, in principle, life on other planets could be detected on a global scale, if it is widely spread and distinguishable from known abiotic spectral signatures. As for the Earth, we already showed that the Vegetation Red Edge which is related to chlorophyll absorption features was larger when continents, versus oceans, were facing the Moon. It proved that an elementary mapping of a planet was even possible. In the frame of the LUCAS (LUmière Cendrée en Antarctique par Spectroscopie) project, the Earthshine has been measured in the Concordia Research Station (Dome C, Antarctica) long enough to observe variations corresponding to different parts of the Earth facing the Moon. An extension of this project, called LUCAS II, would allow long-term observations to detect seasonal variations in the vegetation signal. These data, together with precise measurements of the Earth's albedo, will help to validate a model of global and spectral albedo of our planet.
\end{abstract}

Keywords. Astrobiology, Extraterrestrial life, Earth, Moon, Earthshine, Vegetation

\section{Looking for life in extrasolar planets}

To date, about one thousand exoplanets have been detected and we know a few thousand others are candidates [see the Extrasolar Planets Encyclopaedia published daily on the Internet by Jean Schneider (http://exoplanet.eu/)]. The CoRoT and Kepler satellites have sped up the discovery of new planets. In December 2011, the first Earth-like planet in the habitable zone of a Sun-like star, namely Kepler 22b, has been revealed. Since then, about ten near-Earth sized candidates orbiting in the habitable zone of their host star have been detected. We expect future extremely large instruments and space missions to provide soon the first low-resolution image of an extrasolar planet. Such images will appear as a dot so that detecting life on an unresolved Earth-like extrasolar planet is the next challenge. The spectrum of the light reflected by the planet, when normalized to the parent star spectrum, will gives a reflectance spectrum that mixes information about its surrounding atmosphere and its ground colour. What would the spectrum of an unresolved Earth-like planet look like? Life on an extrasolar planet, if any, probably substantially differs from that on Earth. However, our planet is the only one that is known to provide life. A way to answer this question would consist of measuring the global and spectral albedo of the Earth from a very long distance, typically several 
parsecs. This could be achieved using a space craft traveling through the Solar System and looking back at the Earth, like Voyager 1 in 1990 or Mars Express in 2003.

\section{Earthshine observations}

According to Jean Schneider (1998, private communication), the global spectrum of the Earth can be obtained in another way by measuring the spectral reflectance of the Moon Earthshine, i.e. the light back-scattered by the non-sunlit Moon. A spectrum of the Earthshine directly gives the disk-averaged spectrum of the Earth. Because of the lunar surface roughness, any place of the Earthshine reflects the whole enlighted part of the Earth facing the Moon. As soon as 1912, Arcichovsky (1912) suggested to look at the chlorophyll absorption features in the Earthshine spectrum, to calibrate this photosynthetic pigment in the spectrum of other planets. However, such a detection was impossible at that time. This approach was then completely forgotten and independently rediscovered in 1998. In the Earth reflected spectrum, we shall look for the signature of atmospheric molecules (biogenic products) $\mathrm{O}_{2}, \mathrm{O}_{3}, \mathrm{CH}_{4}, \mathrm{H}_{2} \mathrm{O}, \mathrm{CO}_{2}$ and biological activity. Up to no detection of animal life on Earth in observational conditions of an extrasolar planet has been reported. For instance, the Great Barrier Reef $(2,600 \mathrm{~km})$, which is considered as the world's largest structure composed of living entities, cannot be detected at long distance from its spectral signature. Contrary to Earth vegetation. The best example is the Amazonian forest that covers about $7 \times 10^{6} \mathrm{~km}^{2}$. However, one should keep in mind that in another physical environment, vegetation may take forms and colours that are unfamiliar to us (e.g. Kiang (2008)). We decided to take the Earth as a model. The reflectance of plant canopies is about 9 times higher in the near infrared $(900 \mathrm{~nm})$ than in the red $(660 \mathrm{~nm})$ part of the electromagnetic spectrum. This produces a sharp edge around $700 \mathrm{~nm}$, the so-called Vegetation Red Edge (VRE). Since 2002 (Arnold et al. (2002), Woolf et al. (2002)), several observations of the VRE have been made of the VRE using the Earthshine. Although VRE is only a few percents, this signature that is typical of vegetation is detectable in an integrated (or disk-averaged) Earth spectrum. Arnold et al. (2002) and Hamdani et al. (2006) showed that it was the lowest when an ocean was facing the Moon (1.3\% for the Pacific Ocean) and the highest when continents were present (4\%). In addition the red side $(600-1000 \mathrm{~nm})$ of the Earth reflectance spectrum shows the presence of $\mathrm{O}_{2}$ and $\mathrm{H}_{2} \mathrm{O}$ absorption bands, while the blue side $(320-620 \mathrm{~nm})$ shows the Huggins and Chappuis ozone $\left(\mathrm{O}_{3}\right)$ absorption bands (Hamdani et al. (2006)).

\section{Conditions of Earthshine observations}

As it is well known, at mid- or low-latitude, Earthshine observations are possible during twilight, i.e. just after the sunset or just before the sunrise. Observations are consequently possible only during a short period of time. Roughly speaking, only two enlighted parts of Earth face the Moon during one observation: either the part located at the West of the telescope for evening observations (beginning of the lunar cycle), or the part of Earth located at the East of the telescope for morning observations (last days of the lunar cycle). From an idea of Jean Schneider (2002, private communication), if observations are made from a site located at high latitudes, the Earthshine can be monitored during several hours, about 8 times a year. Near the poles, they can even last 24 hours, i.e. a total nychthemeron. During such a long period, due to the Earth's rotation, different landscapes alternately face the Moon. The Dome C in Antarctica actually offers a perfect spot for that kind of observations. The French-Italian Concordia station (latitude $75^{\circ} \mathrm{S}$, 
altitude $3,250 \mathrm{~m}$ ) which is open to winter-over teams since 2005, provides exceptional conditions for astronomical observations, specially in the infrared. Information about observational conditions obtained after 10 years of site testing can be found in Fossat (2011).

\section{The LUCAS program}

After inquiring about the possibility of observing the Earthshine from Concordia, we designed the LUCAS (LUmière Cendrée en Antarctique par Spectroscopie) experiment, which consists in an instrument dedicated to Earthshine spectroscopic observations in extreme conditions. The first observing campaign has been unsuccessful due to extreme temperatures and atmospheric conditions (problems of thermal insulation). The feedback we got from this campaign was very important to detect, analyze and correct the instrumental weaknesses. During the following winterover campaign, we obtained Moon spectra during all the observational sequences from the winter (June) solstice to the end of observing time of the year 2009. Observations could be carried out during up to 8 contiguous hours. Such a long observational time is impossible at mid- or low-latitude!

\section{The future of Earthshine observations from Antarctica: LUCAS II}

Our goal is to continue the Earthshine observations during at least four years to study the Earth global and spectral albedo. It will allow us to determine its variations throughout the year and to detect the interannual variability. Using the PROSAIL radiative transfer model, we expect to detect seasonal variations of the vegetation signal as a function of the Leaf Area Index (LAI). PROSAIL is the short name of two coupled models that are very popular in the remote sensing community: PROSPECT, a leaf optical properties model (Jacquemoud \& Baret (1990)), and SAIL, a canopy reflectance model (Verhoef (1984)). It could be possible to estimate the average amount of vegetation present on Earth. The interaction with the atmosphere can be taken into account using a radiative transfer model through the atmosphere. The LUCAS II program will reuse part of the instrumentation deployed during LUCAS I. Results will be correlated to satellite measurements and to other measurements of the Earth albedo, in the context of climate change studies. It would be also very interesting to include similar observations made in Northern high latitude in this program, in order to double the number of Earthshine observations. In Antarctica, observations from around the March equinox up to the June solstice will correspond to the last days of the lunar Cycle, i.e. from the last quarter to a few days before the new moon; observations from the June solstice to approximatively the September equinox will correspond to the first days of the lunar cycle, i.e. from a few days after the new moon to the last quarter. Northern high latitudes observations would occur as long, but in opposite configurations.

When the Earthshine cannot be observed, LUCAS II could be used to study stars with rapid variations, like Be stars, the period of which ranges from hours to days. These rapid variations are attributed to non-radial pulsations. Continuous observations over a long period would allow us to detect them. The interest of such observations from Antarctica is exposed in Briot (2005).

\section{Conclusion}

LUCAS is one of the first astronomy research program with spectroscopic observations at Dome C. We measured the Earth vegetation spectrum during several hours, 
detecting variations during the Earth's rotation, as it will be possible in the future for extrasolar planets. The LUCAS II program will extend the observations of the Earthshine from Antarctica during several years, providing very important information on the Earth albedo and variation of vegetation remote sensing. Concerning observations of Earthshine, some geophysical applications also exist, according to the recommendations made by the NASA Navigator Program: "Continued observations of Earthshine are needed to discern diurnal, seasonal, and interannual variations."

The main collaborators of this study are Jérome Berthier (Observatoire de ParisMeudon), Patrick Rocher (Observatoire de Paris-Meudon), Jean Schneider (Observatoire de Paris-Meudon) and the winterover observers in Antarctica: Karim Agabi, Eric Aristidi, Erick Bondoux, Zalpha Challita, Denis Petermann and Cyprien Pouzenc.

\section{References}

Arcichovsky V. M. 1912, Ann. Inst. Polytech. Don Tsar. Alexis (Novotcherkassk), 1(17), 195

Arnold, L., Gillet, S., Lardière, O., Riaud, P., \& Schneider, J., 2002, A 6 A ,392, 231

Briot, D., 2005, Dome C Astronomy and Astrophysics Meeting, EAS Publications Series 14, 275 Fossat E., 2012, arXiv:1101.3210, http://arxiv.org/abs/1101.3210

Hamdani, S., Arnold, L., Foellmi, C., Berthier, J., Billeres, M., Briot, D., François, P., Riaud, P., \& Schneider, J., 2006, A\&A 460, 617

Jacquemoud, S., \& Baret, F., 1990, Remote Sensing of Environment, 34, 75

Kiang, N. Y., 2008, Scientific American, 298, April 1, 12

Verhoef, W., 1984, Remote Sensing of Environment, 16, 125

Woolf, N. J., Smith, P. S., Traub, W. A., \& Jucks, K. W., 2002, ApJ, 574, 430 